\title{
Procedure Guideline for Diuretic Renography in Children 3.0*
}

Barry L. Shulkin ${ }^{1}$, Gerald A. Mandell ${ }^{2}$, Jeffrey A. Cooper ${ }^{3}$, Joe C. Leonard ${ }^{4}$, Massoud Majd ${ }^{5}$, Marguerite T. Parisi ${ }^{6}$, George N. Sfakianakis ${ }^{7}$, Helena R. Balon ${ }^{8}$, and Kevin J. Donohoe ${ }^{9}$

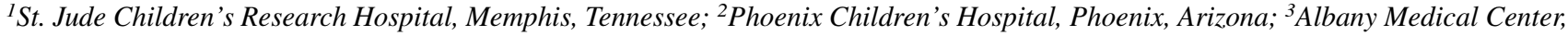
Albany, New York; ${ }^{4}$ Oklahoma Children's Memorial Hospital, Oklahoma City, Oklahoma; ${ }^{5}$ Children's National Medical Center, Washington, DC; ${ }^{6}$ Children's Hospital and Regional Medical Center, Seattle, Washington; ${ }^{7}$ University of Miami School of Medicine, Miami, Florida; ${ }^{8}$ William Beaumont Hospital, Royal Oak, Michigan; and ${ }^{9}$ Beth Israel Deaconess Medical Center, Boston,

Massachusetts

\section{PURPOSE}

The purpose of this guideline is to assist nuclear medicine practitioners in recommending, performing, interpreting, and reporting the results of diuretic renography in children.

\section{BACKGROUND INFORMATION AND DEFINITIONS}

Pelvicaliectasis (distension of the pelvicalyceal system) with or without megaureter (distension of the ureter) is the most common indication for radionuclide evaluation of the kidneys in pediatric patients. Pelvicaliectasis may result from either congenital or acquired etiologies. Included among the causes of pelvicaliectasis are entities such as an obstructed renal pelvis, an obstructed ureter, a duplex renal collecting system, vesicoureteral reflux, bladder abnormalities including neurogenic bladder, bladder outlet obstruction, and infection. Pelvicaliectasis and megaureter can result from obstructive or nonobstructive causes. Obstruction may occur at the level of the ureteropelvic junction, the ureterovesical junction, the posterior/prostatic urethra, or uncommonly in the ureter. Nonobstructive causes include vesicoureteral reflux, nonobstructive pelvicaliectasis or megaureter, prune belly syndrome, and congenital megacalycosis.

Contrast intravenous urography, ultrasonography, and conventional radionuclide renography cannot reliably differentiate obstructive from nonobstructive causes of pelvicaliectasis and megaureter.

The pressure perfusion study (Whitaker test), which measures collecting system pressure while the renal pelvis is

\footnotetext{
Received Aug. 4, 2008; revision accepted Aug. 4, 2008.

For correspondence or reprints contact: Kevin Donohoe, Beth Israel Deaconess Medical Center, 330 Brookline Ave., Boston, MA 02215.

E-mail: kdonohoe@caregroup.harvard.edu

*YOU CAN ACCESS THIS ACTIVITY THROUGH THE SNM WEB SITE (http://www.snm.org/guidelines).

COPYRIGHT (c) 2008 by the Society of Nuclear Medicine, Inc.

DOI: $10.2967 /$ jnmt.108.056622
}

infused with increasing amounts of fluid, is relatively invasive. It may overestimate obstructive phenomena and diagnose obstruction in cases of reduced renal function, when obstruction appears to occur at a flow rate that the kidney with impaired function cannot achieve.

Diuretic renography is a safe and valuable method for the evaluation of renal function and differentiation between obstructive and nonobstructive causes of renal or ureteral dilation.

Hydronephrosis detected in utero may resolve spontaneously and is related to physiologic change during early development. The diagnosis of obstruction often requires sequential scintigraphic examinations.

Injection time for furosemide in relation to tracer injection is indicated by the letter "F." As an example, injection of furosemide $20 \mathrm{~min}$ after tracer injection is indicated as "F + 20."

\section{PROCEDURE}

\section{A. Patient Preparation}

1. Preparation before arrival in the department is usually not necessary. If the patient is not going to receive intravenous fluids, oral hydration is encouraged before arrival and while in the department. Oral fluids in the range recommended for intravenous administration are appropriate (see III A.2.e.).

2. Preparation before injection of the radiopharmaceutical.

a. The procedure is explained to parents and all children old enough to understand. Parents can remain and help with the examination if their presence is beneficial.

b. Continual communication and reassurance with explanation of each step is essential for cooperation and successful intravenous injection of the radiopharmaceutical and catheterization of the bladder. 
c. Oral hydration (volume expansion) may be sufficient in certain situations. Intravenous hydration is more reliable in the diagnosis of questionable cases of urinary obstruction. An indwelling venous catheter may be inserted to maintain sufficient hydration for a good diuretic effect and obviate repeated traumas from multiple percutaneous injections. For the administration of the diuretic at the time of tracer injection (F0), a 21- or 23-gauge butterfly needle is used for the simultaneous injection of the radiopharmaceutical and the diuretic and may be removed after the injection.

d. Bladder catheterization is not always necessary but is suggested if it is necessary to evaluate patients with bladder pathology or in questionable cases; it is also sometimes necessary to catheterize the patient after the study, to evaluate the effect of the urinary bladder. In some cases, the diagnosis of obstruction may be more reliable with bladder or pelvic drainage catheterization. Older children who are not catheterized are requested to void completely before the study.

i. Sterile urethral catheterization should be performed with the largest-sized Foley or feeding catheter that will comfortably pass the meatus (a 2.6-mm-diameter catheter [French 8] for most patients and 1.8-mm-diameter [French 6] for infants). A French 8 feeding catheter may also be used for continual bladder drainage.

ii. Continual drainage by catheterization of the bladder may be required in patients with hydroureter, vesicoureteral reflux, a neuropathic bladder, a small-capacity bladder, a dysfunctional bladder, or posterior urethral valves.

iii. The diuretic effect can be assessed by comparing the volume of urine excreted during the dynamic phase with the volume of urine excreted during the diuretic phase.

e. Hydration or volume expansion, in patients for whom there is no cardiovascular contraindication, is suggested to reduce the incidence of falsepositive findings. Ten to $15 \mathrm{~mL} / \mathrm{kg}$ of one third or greater normal saline (with or without $5 \%$ dextrose) for $30 \mathrm{~min}$ are infused before the diuretic is administered. The slow administration of fluid is continued during the remainder of the study.

f. If the rate of urine flow is low during hydration, a larger amount of fluid (up to $40 \mathrm{~mL} / \mathrm{kg}$ ) can be administered cautiously with careful assessment of volume status (with particular attention to patients who may have renal or cardiac compromise). g. Some laboratories do not use intravenous hydration or catheter bladder drainage for the initial evaluation (particularly in older children) so that kidneys can be evaluated without intervention.

\section{B. Information Pertinent to Performing the Procedure}

1. Awareness of a prenatal history of urinary tract dilation, a history of prior surgery to the urinary tract, and congenital urinary abnormalities (duplex systems, renal fusion, etc.) are important for accurate interpretation of the study.

2. The review of available past radiographic, ultrasound, and radionuclide studies adds to the accuracy of interpretation of the current study.

3. Nonlatex materials should be used in patients prone to latex allergy (e.g., patients with congenital spinal defects and chronic urethral catheterization).

4. An allergy to sulfa drugs may prevent the use of furosemide (cross reactivity between sulfa and furosemide) in a small percentage of patients. Urethral anesthesia with lidocaine should not be used in patients with an allergic history to lidocaine or its derivatives.

\section{Precautions}

1. The examination table is covered with plastic-lined absorbent paper to contain spilled tracer and reduce contamination of the table during drainage and catheterization.

2. Gentle catheterization by a qualified individual can prevent an overly traumatic and painful experience and results in better cooperation during follow-up examinations.

3. Slow, deep breathing and a gentle forward motion of the catheter should be used to relax a spastic external sphincter.

4. An application of urethral anesthesia (3-5 $\mathrm{mL}$ of lidocaine jelly) in the male urethra 2-5 min before catheterization helps decrease discomfort.

5. A Foley balloon is inflated only after the catheter and its balloon are confirmed to be in the bladder. Urine return can be appreciated with the balloon still positioned in the posterior urethra. The balloon must be deflated before removal from the bladder. When a feeding tube is used for bladder drainage, it should not be advanced too far, to avoid coiling and knot formation.

6. Caution should be observed with postural changes because of possible diuresis-induced hypotension.

7. Sudden abdominal or flank pain can arise during acute distension of the pelvicalyceal system in some patients.

8. There is a small risk of catheter-induced trauma and infection.

\section{Radiopharmaceutical}

1. The preferred radiotracer, ${ }^{99 \mathrm{~m}}$ Tc-mercaptoacetyltri-

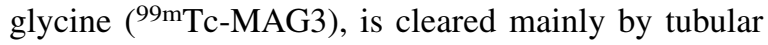


secretion. After about $3 \mathrm{~h}, 90 \%$ of the injected dose can be recovered in the urine. ${ }^{99 \mathrm{~m}} \mathrm{Tc}-\mathrm{MAG} 3 \mathrm{has}$ a high initial renal uptake, providing high kidney-to-background ratios with good temporal resolution. ${ }^{99 \mathrm{~m}} \mathrm{Tc}-\mathrm{MAG} 3$ is recommended for neonatal renography and for visualization of kidneys in patients with compromised renal function. The recommended administered dose is $1.9 \mathrm{MBq}(50 \mu \mathrm{Ci})$ per kilogram of body weight (minimum, $19 \mathrm{MBq}$ [0.5 mCi]). Some laboratories use $37 \mathrm{MBq}(1 \mathrm{mCi})$ as a minimum dose.

2. ${ }^{99} \mathrm{~m} \mathrm{Tc}$-diethylene triamine pentaacetic acid $\left({ }^{99 \mathrm{~m}} \mathrm{Tc}-\right.$ DTPA) is a glomerular agent. The biologic half-life is less than $2.5 \mathrm{~h}$, and $95 \%$ of the administered dose is cleared by $24 \mathrm{~h}$. The recommended administered dose is $3.7 \mathrm{MBq}(100 \mu \mathrm{Ci})$ per kilogram of body weight (minimum, $37 \mathrm{MBq}[1 \mathrm{mCi}]$ ).

Radiation dose estimates are shown in Tables 1 and 2.

\section{E. Image Acquisition}

1. The study is a dynamic renal scan with the patient supine and with the patient's back to the camera. Serial 15- to 30 -s images $(64 \times 64$ or $128 \times 128$ matrix $)$ are acquired for 30-60 min, depending on the technique chosen.

2. A 1-min flow study may be acquired, but the data from the flow should be incorporated into the function study mentioned above. Grouping the data into 2-min images simplifies the visual interpretation.

3. For $\mathrm{F}+20$ or later technique, the prediuretic and postdiuretic phases are acquired either as a single dynamic study starting immediately after the injection of the radiopharmaceutical and continued for 20-30 min after the injection of furosemide or as 2 separate acquisitions.

4. For $\mathrm{F}-15$ and $\mathrm{F}-0$ techniques, dynamic images are acquired for 20-30 min after injection of the radiopharmaceutical.

5. If postdiuresis clearance with the patient supine is poor, additional dynamic images with the patient prone may be obtained. An alternative technique is acquisition of static images before and after the patient is kept upright for $10-15 \mathrm{~min}$.

\section{F. Interventions}

1. The dose of furosemide (Lasix; Sanofi-Aventis) is $1.0 \mathrm{mg} / \mathrm{kg}$, with a usual maximum dose of $40 \mathrm{mg}$. A higher diuretic dose may be necessary in cases of obesity, chronic use of diuretics, or impaired renal function, either unilateral or bilateral.

2. There are 3 different approaches for the time of injection of the diuretic furosemide $(\mathrm{F})$.

a. In the method endorsed by the American Society of Fetal Urology, the diuretic is injected at $20 \mathrm{~min}$ or later after the radiopharmaceutical $(\mathrm{F}+20$ or later), when the entire dilated system is filled with the tracer.

b. In the method developed in Europe, the diuretic is injected $15 \mathrm{~min}$ before the injection of the radiopharmaceutical ( $F-15)$.

c. In the F -0 method, used by some laboratories in the United States and Australia, there is simultaneous injection of the radiopharmaceutical and the diuretic.

\section{G. Processing and Analysis}

1. From the dynamic renal study, careful evaluation of the parenchymal phase reveals renal function, size, and position. Cortical transit time and dilatation of the collecting system may be examined in the excretory phase (initial 2-4 min).

2. Baseline images of the diuretic phase are used for assessment of the diuretic effect.

3. Cinematic viewing of the diuretic phase assesses patient movement. If there is considerable patient motion, regions of interest around the collecting systems of individual frames will have to be compared at various intervals of the study to assess drainage.

4. Regions of interest are drawn around the dilated pelvicalyceal system for curve analysis and calcula-

TABLE 1

Radiation Dose Estimates for ${ }^{99 m T c-M A G 3}{ }^{1}$

\begin{tabular}{|c|c|c|c|c|c|c|}
\hline \multirow[b]{2}{*}{ Organ } & \multicolumn{6}{|c|}{ Estimated radiation dose equivalent (mSv/MBq) } \\
\hline & Newborn & $1-y$-old & 5 -y-old & 10-y-old & $15-y$-old & Adult \\
\hline Kidneys & 0.041 & 0.016 & 0.0097 & 0.0067 & 0.0049 & 0.0041 \\
\hline Ovaries & 0.013 & 0.0069 & 0.0083 & 0.0058 & 0.0085 & 0.0067 \\
\hline Bone surfaces & 0.0053 & 0.0027 & 0.0022 & 0.0015 & 0.0019 & 0.0015 \\
\hline Red marrow & 0.0027 & 0.0014 & 0.0015 & 0.0011 & 0.0015 & 0.0011 \\
\hline Testes & 0.014 & 0.0078 & 0.0082 & 0.0054 & 0.0066 & 0.0046 \\
\hline Urinary bladder wall & 0.37 & 0.15 & 0.17 & 0.11 & 0.17 & 0.14 \\
\hline Effective dose equivalent & 0.032 & 0.014 & 0.015 & 0.010 & 0.015 & 0.012 \\
\hline
\end{tabular}

${ }^{1}$ Stabin and Gelfand. Q J Nucl Med. 1998;42:93-112. 
TABLE 2

Radiation Dose Estimates for ${ }^{99 m}$ Tc-DTPA Injection ${ }^{1}$

\begin{tabular}{|c|c|c|c|c|c|c|}
\hline \multirow[b]{2}{*}{ Organ } & \multicolumn{6}{|c|}{ Estimated radiation dose equivalent (mSv/MBq) } \\
\hline & Newborn & 1-y-old & 5-y-old & $10-y$-old & $15-\mathrm{y}$-old & Adult \\
\hline Kidneys & 0.057 & 0.024 & 0.014 & 0.0095 & 0.0069 & 0.0057 \\
\hline Ovaries & 0.026 & 0.012 & 0.0091 & 0.0061 & 0.0069 & 0.0055 \\
\hline Bone surfaces & 0.027 & 0.013 & 0.0075 & 0.0052 & 0.0040 & 0.0033 \\
\hline Red marrow & 0.019 & 0.0054 & 0.0050 & 0.0034 & 0.0027 & 0.0022 \\
\hline Testes & 0.022 & 0.010 & 0.0077 & 0.0049 & 0.0052 & 0.0038 \\
\hline Urinary bladder wall & 0.19 & 0.079 & 0.086 & 0.058 & 0.097 & 0.077 \\
\hline Effective dose equivalent & 0.034 & 0.015 & 0.012 & 0.0081 & 0.010 & 0.0082 \\
\hline
\end{tabular}

tion of the half-time $\left(\mathrm{T}_{1 / 2}\right)$. One to 2 background regions can also be drawn. The reader is referred to a standardized technique of the "well-tempered" diuretic renogram and recommendations by international consensus report.

5. The diuretic $T_{1 / 2}$ is the time at which the time-activity curve decreases to half its maximal activity. A research study applying $\mathrm{F}-15, \mathrm{~F} 0$, and $\mathrm{F}+20$ indicated that the 3 methods are equivalent for indicating obstruction. They differ in duration (shorter F0) and in patient acceptance and cost (F0 favored). F0 and $\mathrm{F}-15$ also allow evaluation of the renal parenchyma in nonobstructed cases and contribute to the work-up of parenchymal disorders such as focal acute pyelonephritis, HIV nephropathy (AIDS nephropathy), and others.

6. For F +20 studies, residual activity can be reported by estimating the percentage of tracer activity that remains at $20 \mathrm{~min}$ after injection of the diuretic, compared with the activity at the time of diuretic injection. Individual curves from the renal cortices should be produced by carefully assigning the renal cortex away from the collecting system. Such curves can be useful in cases of extrarenal pelvis, nonobstructing pelviectasis, and megaureter and especially in postoperative cases with residual dilatation of the collecting system but no obstruction.

\section{H. Interpretation Criteria}

1. The diuretic effect usually begins within 1-2 min after the administration of the diuretic.

2. In the absence of obstruction, rapid and almost complete washout of the radiotracer occurs before injection of diuretic. However, if function is decreased, there may be slow emptying of the kidneys.

3. Obstructed systems can result in delayed drainage from the collecting system. The amount of activity proximal to the obstruction can also increase over time.
4. With the injection of the diuretic after the radiopharmaceutical $(\mathrm{F}+20$ or later $)$, a $\mathrm{T}_{1 / 2}$ less than $10 \mathrm{~min}$ usually means the absence of obstruction, and a $T_{1 / 2}$ greater than 20 min usually identifies obstruction. A $\mathrm{T}_{1 / 2}$ with a value between 10 and $20 \mathrm{~min}$ is an equivocal result. These $\mathrm{T}_{1 / 2}$ measurements are applicable to neonatal hydronephrosis. The natural history of neonatal hydronephrosis is variable. Drainage may gradually improve or worsen. Therefore, follow-up examinations are usually necessary. These $T_{1 / 2}$ values refer to kidneys with normal or near-normal function. Kidneys with reduced function may have prolonged $\mathrm{T}_{1 / 2}$ values without obstruction.

5. With the injection of the diuretic before the radiopharmaceutical $(\mathrm{F}-15)$, a $\mathrm{T}_{1 / 2}$ greater than $20 \mathrm{~min}$ is compatible with obstruction.

6. With the simultaneous injection of the radiopharmaceutical and furosemide ( $\mathrm{F} 0$ ), a $\mathrm{T}_{1 / 2}$ greater than $20 \mathrm{~min}$ is compatible with obstruction. In cases, however, of extrarenal pelvis, nonobstructing pelviectasis and megaureters of long standing, and particularly postoperative patients with residual dilatation of the collecting system, the possibility of obstruction is studied mainly by observing the cortex and the cortical graphs. When the cortical graphs are normal and the cortices appear empty, then there is no obstruction, even if the curves of the total kidneys have a $T_{1 / 2}$ greater than $20 \mathrm{~min}$. The F0 study should therefore be interpreted not only for the behavior of the collecting system but also for the behavior of the cortex of the kidney in question.

The neonatal kidney is functionally immature. As a result, in the F0 study, neonatal kidneys may show increased residual cortical activity, retaining up to $50 \%$ or more of the peak because of immaturity of the kidneys. Such a phenomenon disappears after the age of $3 \mathrm{mo}$.

This method (F0) applied in the neonate with a dilated collecting system has been observed to provide definitive indications for the existence of obstruction if 
the renogram of the entire kidney is upsloping continuously. Such patients often require surgery. Patients with a downsloping curve usually compensate and do not need an immediate operation, but follow-up. Patients with a horizontal graph need close observation because some of them require surgery.

In acquired obstruction (tumors, renal stones, etc.) complete obstruction is characterized by nonvisualization of the collecting system, associated with a rising curve from the parenchyma; blood flow is often decreased. Partial obstruction is characterized by delayed and persistent visualization of the drainage system and cortical retention of the activity, associated with decreased blood flow. The acute postobstruction/postdecompression image (stunned kidney) shows cortical retention, relatively better flow, faint (if any) visualization of the intrarenal collecting system, but always visualization of the ureter (may need enhancement).

7. The shape of the resulting time-activity curves of the washout study has been used for differentiation of stasis from obstruction. Lack of radiotracer decline after furosemide suggests obstruction, although this can be mimicked by impaired renal function. A brisk decline in activity after diuretic is consistent with stasis without obstruction.

\section{Reporting}

1. The procedure, date of the study, activity and route of administration of the radiopharmaceutical, and a previous study for comparison are included.

2. The history includes symptoms or diagnosis.

3. The technique includes catheter size and type if implemented, amount and kind of intravenous fluid if administered, the imaging sequence, the amount and time of diuretic administration, and the urine volumes before and after the diuretic, if measured.

4. The findings may include renal perfusion, split renal function, transit times, and the $T_{1 / 2}$ of collecting system emptying after the diuretic.

\section{J. Quality Control}

There are no issues of quality control.

\section{K. Sources of Error}

1. Infiltration of the radiopharmaceutical or diuretic may invalidate the results.

2. Insufficient hydration can result in delayed uptake and excretion, simulating poor function, or can demonstrate a normal response in the presence of significant (partial) obstruction.

3. If the diuretic is administered before the maximum distension of the collecting system, the response may not reflect the true physiologic state. However, in the F0 method, the cortex empties appropriately, and this observation compensates for this phenomenon.
4. Poor renal function from prolonged severe obstruction can result in slow tracer accumulation in the dilated collecting system and result in difficulty in interpretation of the diuretic phase. The F0 study provides additional help by indicating a normal emptying cortex in cases of no obstruction. In cases of obstruction, the cortex shows prolonged retention of the activity.

5. A large, unobstructed collecting system with relatively good renal function can exhibit slow drainage of the radiotracer (prolonged $\mathrm{T}_{1 / 2}$ ). The $\mathrm{F}_{0}$ method indicates normal emptying of the cortex.

6. When the obstruction is at both the pelvicalyceal and the ureterovesical junctions, detection of the ureterovesical junction obstruction may be difficult.

7. Patient movement may invalidate curve analysis.

8. Urinary systems considered normal in the prediuretic phase may not be evaluated for postdiuresis drainage. A prolonged $T_{1 / 2}$ can be obtained because of the relatively small amount of residual activity in the collecting system to respond to the diuretic challenge. With the F0 approach, in rare cases the use of the diuretic has uncovered borderline obstructions associated with normal baseline studies in symptomatic patients (pain after much drinking).

\section{ISSUES REQUIRING FURTHER CLARIFICATION}

A. The calculation method of the postdiuresis drainage is variable, but a standardized technique is available in the literature.

B. The curve analysis has been questioned because of poor correlation with pressure perfusion studies in children.

C. The results of the alternative method of simultaneous injection of the radiopharmaceutical and diuretic remain to be validated. However, in some laboratories the method provides valuable and accurate diagnostic and prognostic information noninvasively and quickly.

\section{CONCISE BIBLIOGRAPHY}

A. Bird VG, Gomez-Marin O, Leveillee RJ, Sfakianakis GN, Rivas LA, Amendola MA. A comparison of unenhanced helical computerized tomography findings and renal obstruction determined by furosemide 99m-technetium mercaptoacetyl-triglycine diuretic scintirenography for patients with acute renal colic. J Urol. 2002;167:1597-1603.

B. Conway JJ. Radionuclide cystography. In: Tauxe WN, Dubovsky EV, eds. Nuclear Medicine in Clinical Urology and Nephrology. East Norwalk, CT: Appleton, Century and Crofts; 1985:305-320.

C. Conway JJ. "Well-tempered" diuresis renography: its historical development, physiological and technical 
pitfalls, and standardized technique protocol. Semin Nucl Med. 1992;22:74-84.

D. Donoso G, Kuyvenhoven JD, Ham H, Piepsz A. 99m Tc-MAG3 diuretic renography in children: a comparison between F0 and F+20. Nucl Med Commun. 2003;24:1189-1193.

E. Eskild-Jensen A, Gordon I, Piepsz A, Frokiaer J. Congenital unilateral hydronephrosis: a review of the impact of diuretic renography on clinical treatment. J Urol. 2005;173:1471-1476.

F. Foda MM, Garfield CT, Matzinger M, et al. A prospective randomized trial comparing 2 diuresis renography techniques for evaluation of suspected upper urinary tract obstruction in children. J Urol. 1998;159:1691-1693.

G. Houle AM, Cheikhelard A, Barrieras D, Rivest MC, Gaudreault V. Impact of early screening for reflux in siblings on the detection of renal damage. $\mathrm{Br} \mathrm{J} \mathrm{Urol}$ Int. 2004;94:123-125.

H. Jung HS, Chung YA, Kim EN, et al. Influence of hydration status in normal subjects: fractional analysis of parameters of Tc-99m DTPA and Tc-99m MAG3 renography. Ann Nucl Med. 2005;19:1-7.

I. Kass EJ, Majd M. Evaluation and management of the upper urinary tract obstruction in infancy and child hood. Urol Clin North Am. 1985;12:122-141.

J. Kuyvenhoven JD, Ham HR, Piepsz A. The estimation of renal transit using renography: our opinion. $\mathrm{Nucl}$ Med Commun. 2004;25:1223-1231.

K. Liu Y, Ghesani NV, Skurnick JH, Zuckier LS. The $\mathrm{F}+0$ protocol for diuretic renography results in fewer interrupted studies due to voiding than the F 15 protocol. J Nucl Med. 2005;46:1317-1320.

L. Meller ST, Eckstein HB. Renal scintigraphy: quantitative assessment of upper urinary tract dilatation in children. J Pediatr Surg. 1981;16:123-126.

M. Piepsz A, Ham HR. Pediatric applications of renal nuclear medicine. Semin Nucl Med. 2006;36:16-35.

N. Piepsz A, Ismaili K, Hall M, Collier F, Tondeur M, Ham H. How to interpret a deterioration of split function. Eur Urol. 2005;47:686-690.

O. Prigent A, Cosgriff P, Gates GF, et al. Consensus report on quality control of quantitative measurements of renal function obtained from the renogram: International Consensus Committee from the Scientific Committee of Radionuclides in Nephrourology. Semin Nucl Med. 1999;29:146-159.

P. Senac MO, Miller JH, Stanley P. Evaluation of obstructive uropathy in children: radionuclide renography versus the Whitaker test. AJR. 1984;143: 11-15.

Q. Sfakianaki E, Panagakos GM, Rodriquez JA, Georgiou MF, Leveilee RJ, Sfakianakis GN. Evaluation of obstruction in patients with extrarenal pelvis using time zero diuretic renography [abstract]. J Nucl Med. 2003;44(suppl):359P.
R. Sfakianakis GN, Carmona AJ, Sharma A, et al. Diuretic MAG3 scintirenography in children with HIV nephropathy: diffuse parenchymal dysfunction. J Nucl Med. 2000;41:1037-1042.

S. Sfakianakis GN, Sfakianakis E. Renal scintigraphy in infants and children. Urology. 2001;57:11671177.

T. Sfakianakis GN, Vensel EE, Tapia-Palacios M, et al. The value of MAG3-Fo diuretic renography in predicting the need for surgery in the neonate with uretero-pelvic junction obstruction [abstract]. $\mathrm{J} \mathrm{Nucl}$ Med. 2001;42(suppl):88P-89P.

U. Shokeir AA, El-Sherbiny MT, Gad HM, et al. Postnatal unilateral pelviureteral junction obstruction: impact of pyeloplasty and conservative management on renal function. Urology. 2005;65:980-985.

V. Stabin MG, Gelfand MJ. Dosimetry of pediatric nuclear medicine procedures. Q J Nucl Med. 1998;42:93-112.

W. Vlajkovic M, Ilic S, Rajic M, Petronijevic V, Bubanj T, Artiko V. Diuresis renal scintigraphy " $\mathrm{F}-0$ " in diagnosing of upper urinary tract obstruction in children: the clinical significance. Nucl Med Rev. 2005:8: 21-27.

X. Wackman J, Brewer E, Gelfand MJ, et al. Low grade pelviureteric obstruction with normal diuretic renography. Br J Urol. 1986;58:364-367.

Y. Whitaker RH, Buxton TMS. A comparison of pressure flow studies and renography in equivocal upper tract obstruction. J Urol. 1986;131:446-449.

\section{DISCLAIMER}

The SNM has written and approved this Procedure Guideline as an educational tool designed to promote the costeffective use of high-quality nuclear medicine procedures in medical practice or in the conduct of research and to assist practitioners in providing appropriate care for patients. The Procedure Guideline should not be deemed inclusive of all proper procedures or exclusive of other procedures reasonably directed to obtaining the same results. The guidelines are neither inflexible rules nor requirements of practice and are not intended nor should they be used to establish a legal standard of care. For these reasons, the SNM cautions against the use of this Procedure Guideline in litigation in which the clinical decisions of a practitioner are called into question.

The ultimate judgment about the propriety of any specific procedure or course of action must be made by the physician when considering the circumstances presented. Therefore, an approach that differs from the Procedure Guideline is not necessarily below the standard of care. A conscientious practitioner may responsibly adopt a course of action different from that set forth in the Procedure Guideline when, in his or her reasonable judgment, that course of action is indicated by the condition of the patient, limitations on available resources, or advances in knowl- 
edge or technology subsequent to publication of the Procedure Guideline.

All that should be expected is that the practitioner will follow a reasonable course of action based on current knowledge, available resources, and the needs of the patient to deliver effective and safe medical care. The sole purpose of this Procedure Guideline is to assist practitioners in achieving this objective.
Advances in medicine occur at a rapid rate. The date of a Procedure Guideline should always be considered in determining its current applicability.

\section{APPROVAL}

This Procedure Guideline was approved by the Board of Directors of the SNM on April 15, 2007. 